\title{
Definition of Low Earth Orbit slotting architectures using 2D lattice flower constellations
}

\author{
David Arnas $^{\mathrm{a}, *}$, Miles Lifson ${ }^{\mathrm{a}}$, Richard Linares ${ }^{\mathrm{a}}$, Martín E. Avendaño ${ }^{\mathrm{b}}$ \\ a Massachusetts Institute of Technology, Cambridge, MA 02139, USA \\ ${ }^{\mathrm{b}}$ CUD-AGM (Zaragoza), Crtra Huesca s/n, Zaragoza 50090, Spain
}

Received 27 January 2020; received in revised form 7 April 2020; accepted 11 April 2020

\begin{abstract}
This work proposes the use of 2D Lattice Flower Constellations (2D-LFCs) to facilitate the design of a Low Earth Orbit (LEO) slotting system to avoid collisions between compliant satellites and to optimize the available orbital volume. Specifically, this manuscript proposes the use of concentric orbital shells of admissible "slots" with stacked intersecting orbits that preserve a minimum separation distance between satellites at all times. The problem is formulated in mathematical terms and three approaches are explored: random constellations, single 2D-LFCs, and unions of 2D-LFCs. Each approach is evaluated in terms of several metrics including capacity, Earth coverage, orbits per shell, and symmetries. Additionally, a rough estimate for the capacity of LEO is generated, subject to certain minimum separation and station-keeping assumptions, and several trade-offs are identified to guide policy-makers interested in the adoption of a LEO slotting scheme for space traffic management.
\end{abstract}

(C) 2020 COSPAR. Published by Elsevier Ltd. All rights reserved.

Keywords: Space mechanics; Satellite constellations; Space traffic management; Orbit design; Orbital slotting

\section{Introduction}

SpaceX, OneWeb, Telesat, and other companies are currently planning mega-constellations for space-based internet connectivity and other applications that would significantly increase the number of on-orbit active satellites across a variety of altitudes (SpaceX, 2019; OneWeb, 2019; Telesat, 2019; Portillo et al., 2019; Alary et al., 2018). As these and other actors seek to make more intensive use of the global space commons, there is growing need to characterize the fundamental limits to the capacity of Low Earth Orbit (LEO), particularly in high-demand

\footnotetext{
* Corresponding author.
}

E-mail addresses: arnas@mit.edu (D. Arnas),mlifson@mit.edu (M. Lifson), linaresr@mit.edu (R. Linares), avendano@unizar.es (M.E. Avendaño). orbits and altitudes, and minimize the extent to which use by one space actor hampers use by other actors.

This manuscript contributes to this goal by proposing a slotting mechanism for LEO, based on the Flower Constellation (FC) Theory (Mortari et al., 2004; Mortari and Wilkins, 2008; Wilkins and Mortari, 2008). In particular, the 2D Lattice Flower Constellation (2D-LFC) (Avendaño et al., 2013) formulation is used to define sets of admissible satellite locations or "slots" while ensuring a minimum separation distance between all slots at all times. This slotting system would greatly reduce the risk of satellite vs. satellite collisions (eliminating it for conjunctions involving compliant satellites), decrease the analysis and coordination burden associated with such conjunctions, and potentially allow for the safe co-location of spacecraft controlled by different operators at the same altitudes as mega-constellations. Additionally, this proposed solution is compared with other approaches in order 
to determine the trade-offs of different design methodologies.

Access to Earth orbit belongs to a class of goods known as common pool resources (CPRs) (Weeden and Chow, 2012). These resources are characterized by the ability of multiple actors to make use of them and the fact that one actor's use degrades that resource for others. Terrestrial examples of CPRs include public roads, aquifers, and fisheries (Gardner et al., 1990). In the case of the space environment, costs of CPR use include increased risk of physical collision and spectral interference, as well as limitations on use of particular physical regions and spectrum bands already in use by others. A CPR dilemma can be distinguished from a CPR situation by the presence of two additional conditions: (1) less-than optimal outcomes as measured by those using the resource and (2) the existence of at least one potential strategy that is both feasible and pareto-improving (Gardner et al., 1990, 6-7). Managing CPR dilemmas to ensure sustainability is a classic public policy problem.

Assignment systems (including slotting mechanisms) are a standard CPR management strategy. An assignment system enforces coordination on those using the CPR through top-down allocation of the right to use the resource subject to certain conditions, with the objective of preserving usability for all. ${ }^{1}$ Within the space domain, such systems exist and are widely used for the allocation of electromagnetic spectrum for communications and remote sensing at both national and international levels. Most notably, the International Telecommunications Union operates a system to allocate spectrum for geostationary orbital slots to prevent harmful signal interference (Jakhu, 2017).

The International Academy of Astronautics' 2018 report on Space Traffic Management (STM) describes pathways towards the development of both a "technical STM system" and a "regulatory STM regime" and sketches out the elements necessary for either a top-down or bottom-up approach to STM (Alary et al., 2018). A LEO slotting mechanism is not mentioned explicitly, but could be implemented under either approach. With a bottom-up strategy, a slotting system could see admissible slot locations defined on a voluntary consensus-basis, and actors build norms for satellite deployment into slots and station-keeping adequate to stay within the relevant control-volumes. With a top-down approach, slots might be allocated by an international intergovernmental organization.

Because LEO is so much larger than Geostationary Earth Orbit (GEO), there has historically been less concern about orbital access in LEO (although debris continues to be a major concern and particular regions of LEO, including Sun-Synchronous Orbits (SSOs), are increasingly congested). Nevertheless, the emergence of multiple proposed

\footnotetext{
${ }^{1}$ Other systems are also possible. For instance, coordination can evolve iteratively and organically by cooperation and voluntary modifications to use strategies by individual CPR users (Gardner et al., 1990, 9-10).
}

mega-constellations, each occupying a significant altitude band on a potentially exclusive basis, has sparked discussions about apportionment of orbital volume in LEO in a manner that preserves access for emerging actors, both states and commercial entrants. In 2019, representatives of OneWeb, Iridium, and DigitalGlobe, a Maxar Technologies company, authored an op-ed for SpaceNews that, among other recommendations, called for avoiding overlapping altitudes for large constellations. The European Space Agency has conducted work to develop a space capacity metric to understand the impact of a particular mission on the overall LEO environment (Letizia et al., 2019).

The LEO slotting problem is considerably more complex than GEO due to the need to accommodate multiple altitudes, eccentricities, and overlapping orbits. The limited work to date on LEO slotting has primarily focused on SSO. In 2007, the International Space University Summer Session Program produced a set of technical traffic and environmental rules that included a proposal for zoning of SSOs (Anilkumar et al., 2007, 22-27) that was further developed in Weeden and Shortt (2008). The authors mention the possibility of using specially-defined timing to allow for overlapping orbits, but do not present a technical system to determine such slotting. Later, Bilimoria and Krieger (2011) presented a slot architecture for SSO, including a phasing rule to preserve minimum separation at near-polar crossing points and a proposal for slot sizing and dead-band control to preserve satellite separation in the presence of various perturbations. In his Master's thesis, Watson (2012) conducted analysis to characterize the relative motions of sun-synchronous satellites about a given slot location and developed an architecture and tool to generate SSO slots and control-volume geometry based on user-selected values for parameters for slot sizing including semi-major axis, time between station-keeping maneuvers, altitude loss rate, and relative motion oscillation amplitude. In a Master's thesis, Noyes (2013) built on Watson's work with further relative motion analysis to detect and analyse satellite periods of conformance for different altitudes and ballistic coefficients, as well as characterize the impact of an SSO slotting architecture on collisions and debris generation using an evolutionary debris model.

This work builds on these efforts to present a generalized approach to designing orbits that preserve minimum separation between participating satellites at all times, expanding consideration beyond SSO. However, the nature of the approach requires certain decisions regarding how to allocate orbits, managing concerns like orbital density, coverage, and the prioritization of certain orbital inclinations (Arnas et al., 2020). For the purpose of this paper, we focus on the technical design of a potential slotting system and leave questions such as allocation, orbital configuration, and other value trade-offs to subsequent work.

This manuscript makes four main contributions. First, it provides a mathematical formulation of the LEO slotting 
problem. Second, it describes three approaches to LEO slot generation and the resultant maximum capacity per shell. Third, it defines four metrics for evaluating potential slot configurations. Fourth, it demonstrates how the number of admissible slots per shell can be used as a metric for LEO capacity, both to characterize overall capacity and to help understand the trade-offs between different decisions regarding spacecraft placement.

This manuscript is organized as follows. Section 2 includes a summary of the FC Theory and previous results applying FC theory to this problem. In Section 3, we propose what we believe is a precise, but approach-agnostic definition for the problem of generating sets of LEO slots free from self-conjunctions. We then narrow the problem to a particular altitude shell and describe what constitutes a valid solution to this sub-problem. Additionally, we provide an analytical tool to determine the minimum distance between satellites when dealing with constellations defined at the same altitude and composed of only circular orbits. In Section 4, several versions of potential orbital slotting systems are considered including random constellations, single 2D-LFCs, and several forms of unions 2D-LFCs. These are experimentally evaluated using computer simulation against a set of four metrics: capacity, Earth coverage, orbits per shell, and symmetries. Section 5 describes several implications for a policy-maker or system architect tasked with selecting slot generation strategies across various orbital shells. Subject to a set of assumptions for minimum separation distance and other factors, we estimate a capacity of admissible slots in LEO under a notional design strategy.

\section{Preliminaries}

\subsection{Flower constellation theory}

The Flower Constellation Theory is a set of constellation design models that focuses on the generation of uniform distributions of satellites in the space. Flower Constellation theory has been demonstrated in the literature to design constellations for a wide variety of applications including Earth observation, Earth observation with intersatellite links, telecommunication, telemedicine, global navigation satellite service design, and Lunar and Martian constellations (Marzano et al., 2009; Lee and Mortari, 2017; Mortari et al., 2011; De Sanctis et al., 2008; Park et al., 2005; Mortari et al., 2013; McManus and Schaub, 2016; De Sanctis et al., 2007). The theory started with a design model to distribute satellites along a relative trajectory that is defined in an arbitrary rotating frame of reference (Mortari et al., 2004). This formulation allowed the generation of completely uniform distributions that presented a series of patterns that were repeated over the constellation.

Later, in order to simplify the formulation and extend the design possibilities, the 2-D Lattice Flower Constellations (2D-LFC) (Avendaño et al., 2013) were introduced.
The idea behind this methodology is to generate completely uniform distributions of satellites using, as distribution variables, the right ascension of the ascending node $\Omega$, and the mean anomaly $M$, while the semi-major axis of the orbits $a$, the inclination $i$, the eccentricity $e$, and the argument of perigee $\omega$ are fixed for all the satellites in the configuration. Accordingly, it is possible to define the constellation configuration using just three independent integer parameters: the number of orbital planes, $N_{o}$, the number of satellites per orbit, $N_{s o}$, and the configuration number, $N_{c}$ (phasing parameter). In particular, the relative distribution of each satellite of the constellation $\left(\Omega_{i j}\right.$ and $\left.M_{i j}\right)$ is provided by the following equation:

$$
\left[\begin{array}{cc}
N_{o} & 0 \\
N_{c} & N_{s o}
\end{array}\right]\left\{\begin{array}{l}
\Omega_{i j} \\
M_{i j}
\end{array}\right\}=2 \pi\left\{\begin{array}{l}
i-1 \\
j-1
\end{array}\right\}
$$

where $i=1, \cdots, N_{o}, j=1, \cdots, N_{s o}$ names the $j$-th satellite on the $i$-th orbital plane of the constellation. In order to avoid duplicates in the formulation, the configuration number is defined in the range $N_{c} \in\left[0, N_{o}-1\right]$.

All satellite distributions generated using this formulation have the property of perfect uniformity, that is, the relative distribution of the constellation is fixed no matter which satellite is selected as a reference. This also means that the number of symmetries in the configuration is maximized. This property can be used, for instance, to reduce the computational effort to assess the minimum distance between satellite pairs. In particular, it is sufficient to evaluate the minimum distance between the first satellite $\left[\Omega_{11}, M_{11}\right]$ of the configuration, and all the other satellites. This implies $\left(N_{o} N_{s o}-1\right)$ total checks instead of $N_{o} N_{s o}\left(N_{o} N_{s o}-1\right) / 2$ check of a general distribution. This greatly simplifies the optimization process to find configurations that avoid satellite conjunctions.

Lattice Flower Constellations include, as a subset, the most widespread satellite constellation designs, including Walker Delta Pattern Constellations (Walker, 1984), Streets of Coverage (Luders, 1961), Draim Constellations (Draim, 1987), and Dufour Constellations (Dufour, 2003). In fact, the 2D-LFC methodology is able to generate all the possible uniform distributions performed in the right ascension of the ascending node and the mean anomaly (see Theorems 4, 5, 6 and 7 in Arnas (2018)). This provides a powerful tool to study all possible uniform distributions of satellites with a unified theory. Therefore, we use 2DLFCs as a basis to propose solutions for LEO spacecraft slotting.

\subsection{Non-self-intersecting 2-D lattice flower constellations}

A first approach to design mega-constellations using 2D-LFC was introduced in Lee et al. (2015). The idea proposed in that work was to generate constellations made of circular orbits belonging to a unique repeating relative trajectory that presents no self-intersections. This relative trajectory was defined in a fictitious frame of reference that 
was rotating at a constant speed about the axis of rotation of the Earth. That way, a relation can be defined between this rotating frame and the inertial frame of reference:

$N_{d} T_{d}=N_{p} T_{p}$

where $T_{d}$ is the rotating period about the axis of rotation of the Earth of a fictitious rotating frame of reference, $T_{p}$ is the revolution period of the satellite in the inertial frame, and $N_{d}$ and $N_{p}$ are the minimum number of complete revolutions of the fictitious rotating frame and the orbits respectively until the dynamic is repeated in both the inertial and the rotating frames of reference. Then, under this framework, it is guaranteed that no satellite conjunctions will occur since all satellites follow the same relative non self-intersecting path at any instant. This facilitates a focus on maximizing the minimum distance between satellites.

In general, the 2D-LFC design formulation generates constellations that are distributed in different repeating relative trajectories. However, it is possible to use this formulation to generate constellations belonging to the same relative trajectory by imposing the following condition:

$$
\left[\begin{array}{cc}
N_{o} & 0 \\
N_{c} & N_{s o} \\
N_{p} & N_{d}
\end{array}\right]\left\{\begin{array}{l}
\Omega_{i j} \\
M_{i j}
\end{array}\right\}=\left\{\begin{array}{l}
0 \\
0 \\
0
\end{array}\right\} \bmod 2 \pi
$$

Moreover, in order to assure the non self-intersection of the relative trajectory, two additional constraints are required. In particular, these constraints are provided by the following Theorem (Lee et al., 2015) for prograde orbits: "The relative trajectory of a satellite has no selfintersections if and only if the inclination $i<\pi / 2$ and:

$$
\begin{aligned}
& \text { if } N_{d}=N_{p}-1 \\
& \rightarrow i \max \left[\cos ^{-1}\left(\frac{\tan \left(\phi \frac{N_{p}}{N_{d}}-\frac{\pi}{2 N_{p}}\right)}{\tan (\phi)}\right)\right] \forall \phi \in[0, \pi / 2] ; \\
& \quad \text { if } N_{d}=N_{p}+1 \rightarrow i \cos ^{-1}\left(N_{p} / N_{d}\right) \text {. }
\end{aligned}
$$

This means that, in general, the range of possible inclinations is very limited. Following this process, Lee et al. (2015) were able to generate constellations of 400 satellites. In this work we will overcome this limitation and show other possibilities that 2D-LFCs can provide, both increasing the number of satellites and the range of available inclinations.

\section{Mathematical formulation of the LEO slotting problem}

This section defines the slotting problem and admissible solutions in mathematical terms. In doing so, we hope to formulate the problem precisely and in a manner that allows for direct comparison and evaluation of the various approaches discussed here, as well as for comparison with solutions that might be generated elsewhere in the community.

\subsection{The orbital slot definition problem}

We define a "slot" as a three-dimensional, moving, rotating, and possibly morphing region $S=S(t)$ of the space. The distance between two slots is defined as the minimum distance that, at any instant of time, a point in one of the slots is from a point of the other.

$$
\operatorname{dist}\left(S_{1}, S_{2}\right)=\min \left\{\|p-q\|: p \in S_{1}(t), q \in S_{2}(t), t \in \mathbb{R}\right\}
$$

We want to produce a list of slots $S_{1}, \ldots, S_{n}$ in the LEO space (the spherical shell of space located below $2000 \mathrm{~km}$ of altitude) in such a way that:

$\left(P_{1}\right)$ The slots do not overlap at any time. Moreover, they are separated at any time by a given minimum distance $d_{\text {slot }}>0$.

$\operatorname{dist}\left(S_{i}, S_{j}\right) \geqslant d_{\text {slot }} \quad \forall i \neq j$

This requirement ensures that two adequately sized slots remain separated by a certain minimum distance sufficient to ensure that a satellite maintaining itself within $S_{i}$ will never collide with a satellite maintaining itself in $S_{j}$.

$\left(P_{2}\right)$ It is feasible, control-wise, to keep a satellite contained within any of the slots. That is, there is no need to perform expensive maneuvering to prevent the satellite from leaving a slot, even in presence of orbital perturbations such as the atmospheric drag, the solar radiation pressure or the $J_{2}$ effect. Naturally, there is no point in defining a set of slots within which it is prohibitively costly for satellites to maintain their positions, or that imposes a significant restriction on their propulsion systems.

$\left(P_{3}\right)$ If a hypothetical mission were possible using a set of satellites in LEO orbits, then it should also be possible using a similar (or at least not much larger) number of satellites spread across a set of slots that avoid self-conjunctions. This requirement stems from the idea that a LEO slotting system should be minimally burdensome.

Note that the minimum distance $d_{\text {slot }}$ required between satellites, as well as the maximum station-keeping cost per satellite per day, are design parameters that should be clearly stated in any proposed solution.

A successful solution of the problem must describe the position, orientation, and shape of the slots $S_{1}, \ldots, S_{n}$ as a function of time, and provide a proof that those regions satisfy condition $\left(P_{1}\right)$. Additionally, to evaluate whether a proposed solution satisfies $\left(P_{2}\right)$, the initial conditions (for instance, position and velocity) of $n$ satellites must be provided, each moving within its corresponding slot, as well as a computer simulation of the motion under a chosen perturbation model during several periods of time. This should include a study on the frequency of the required station-keeping maneuvers and their cost. 
At the moment, there is not a clear method to properly evaluate condition $\left(P_{3}\right)$, but we would expect a candidate solution to demonstrate analysis showing that the proposed slotting can accommodate various mission archetypes and at what (if any) additional cost. In that sense, a reference set of design missions would be helpful to enable comparative evaluation of different slotting concepts.

\subsection{Proposed approach A: a mega-constellation}

Since any solution of the problem must include the trajectories of the satellites moving inside the slots (and any station-keeping maneuvering required), it makes sense to design the slots starting from these trajectories. More precisely, we envision that a potential solution can be obtained by the following procedure:

$\left(A_{1}\right)$ Find a constellation of satellites $s_{1}, \ldots, s_{n}$, under the keplerian $/ J_{2}$ model, moving within the LEO region in such a way that they are always separated by at least a distance $d_{\text {const }}$.

$\left(A_{2}\right)$ Study the maximum deviation $d_{\text {pert }}$ that any satellite can experience from its nominal trajectory in a given reference time $t_{\text {pert }}$ due to all non-keplerian $/ J_{2}$ forces included in the model.

$\left(A_{3}\right)$ Define the slots as $S_{1}, \ldots, S_{n}$ as spheres with center at the position of the satellites $s_{1}, \ldots, s_{n}$ and radius $d_{p e r t}$.

If the value of $d_{\text {const }}$ chosen in $\left(S_{1}\right)$ satisfies $d_{\text {const }} \geqslant 2 d_{\text {pert }}+d_{\text {slot }}$, then it can be easily shown that the slotting strategy defined satisfies $\left(P_{1}\right)$. The minimum frequency of the station-keeping maneuvers is controlled by the parameter $t_{\text {pert }}$, which can be defined by the designer as a function of the known orbital perturbations, but the cost of each maneuver depends mostly on $d_{\text {pert }}$, which is a derived quantity. Finding the right balance between frequency of maneuvering and cost of each maneuvers should be included as part of the analysis to be done in $\left(A_{2}\right)$. No special provisions are made concerning $\left(P_{3}\right)$. However, we believe that a large value of $n$ and certain regularity in the design of the constellation in $\left(A_{1}\right)$ would be enough to comply with $\left(P_{3}\right)$.

Of course, many improvements can be made to the approach above. One of the most promising options comes from the shape of the slots themselves. While the idea of using spheres is neat and reduces the mathematical proofs to the triangle inequality, computer simulations show that effects of the non-keplerian $/ J_{2}$ forces in the cross-track direction are lower than in the along-track direction. Accordingly, more slots could potentially be accommodated for a given level of station-keeping requirements if spherical slots were replaced with a shape more elongated in the along-track direction.

Finally, the decision to include the $J_{2}$ effect in $\left(A_{1}\right)$ is due to the fact that at LEO altitudes it is almost impossible to compensate the effects of this perturbation with stationkeeping maneuvers. This implies that for usual values of $t_{\text {pert }}$ in $\left(A_{2}\right)$, we would get a huge value of $d_{\text {pert }}$.

\subsection{Proposed approach B: a multi-layer megaconstellation}

As a first study of step $\left(A_{1}\right)$, we propose a layer-by-layer approach instead of trying to build the entire constellation at once. The vast majority of active satellites use circular and near circular orbits, and the forces mentioned above change significantly in magnitude depending on the altitude. While this approach does restrict the solution to having only circular orbits, it is possible to introduce, using the revolution time compatibility between orbits, a small number of elliptical orbits on demand at the cost of allocating several slots to guarantee that no collisions can occur. Another positive aspect of this type of solutions is that it is possible to open a launching window from any point on Earth, every $1 \mathrm{~cm}\left(N_{1}, \ldots, N_{n}\right)$ days (where $N_{i}$ is the number of days that each satellite requires to repeat its dynamic from the Earth Centered Earth Fixed frame of reference), by leaving empty a subset of specific slots in each layer. Whether this least common multiple can be made small enough to be practical is still under analysis.

With a layer-by-layer approach, the final constellation is a collection of more simple constellations, each corresponding to a single altitude. In particular, each of these simpler constellations has all its satellites moving in circular orbits of the same radius. With this new idea in mind, the recipe above can be reformulated as follows:

$\left(B_{1}\right)$ Choose an altitude $h$ within LEO and find a constellation of satellites $s_{1}, \ldots, s_{n}$, all moving in circular orbits at that altitude in the keplerian $/ J_{2}$ model, in such a way that the distance between satellites is at least $d_{\text {const }}$ at any time.

$\left(B_{2}\right)$ Estimate the maximum deviation in along-track $d_{\text {along }}$ and cross-track $d_{\text {across }}$ that any satellite at altitude $h$ can experience due to non-conservative forces from its nominal orbit in a given fixed time $t_{\text {pert }}$.

$\left(B_{3}\right)$ Define the slots $S_{1}, \ldots, S_{n}$ as the three-dimensional regions of space delimited by altitudes $h-d_{\text {across }}$ and $h+d_{\text {across }}$, and the spheres of radius $d_{\text {along }}$ centered at the corresponding satellite.

$\left(B_{4}\right)$ Return to $\left(B_{1}\right)$ and choose another altitude $h$ in such a way that the selected altitude also guarantees the safety of the configuration.

The collection of all the slots produced by the shown process satisfies conditions $\left(P_{1}\right)$ and $\left(P_{2}\right)$ if some care is taken when choosing the different altitudes. More precisely, within each layer we need to impose the condition $d_{\text {const }} \geqslant 2 d_{\text {along }}+d_{\min }$, and between any two consecutive layers, at altitudes $h^{(1)}$ and $h^{(2)}$, we need $\left|h^{(1)}-h^{(2)}\right| \geqslant d_{\text {min }}+d_{\text {cross }}^{(1)}+d_{\text {cross }}^{(2)}$. These two conditions are sufficient to guarantee that the slotting produced is 
valid. Nevertheless, validity alone is insufficient to determine whether a particular solution obtained for the main problem using approach $\mathrm{B}$ is useful or optimal since that answer will depend on the precise missions under study and the characteristics of future spacecrafts and payloads.

The $J_{2}$ effect must be included in $\left(B_{1}\right)$ if station-keeping maneuvering is to be kept within reasonable values. However, a simpler approach based on a keplerian model is possible when dealing with a layered slotting architecture. The idea is that if the constellation used in $\left(B_{1}\right)$ has all satellites at the same inclination (as in a 2D-LFC), and the distances between satellites is at least $d_{\text {const }}$ as required, then including $J_{2}$ in the model will not change the relative distribution of the constellation. In particular, the rate of precession of all the satellites will be the same, hence relative distances will be preserved. The perfectly spherical shells that we would have in the keplerian model will become slightly deformed, but the deformation is in the same direction in all the shells, so no correction to the separation between layers is needed.

\subsection{Criteria to evaluate and compare solutions of the problem}

It is relatively easy to produce constellations of satellites that satisfy the requirements of the problem. However, not all such constellations are actually useful. For instance, a constellation with all satellites in a single inertial orbit makes little sense, since the practical applications are very limited and the overall capacity is much smaller than could otherwise be achieved. Therefore, in order to define a common framework of design and study of this kind of configurations, we propose the use of four characteristics to help distinguish between the trade-offs of various approaches to solution generation. These characteristics are:

1. Capacity (number of slots per shell),

2. Earth coverage,

3. Orbits per shell,

4. Symmetries.

The first two criteria give quantities that should generally be maximized. The third quantity is trickier: the more inertial orbits the constellation has, the more expensive it would be to deploy (or at least time-consuming if nodal precession is used to space out inertial orbits). On the other hand, it makes little sense to put all satellites in the same inertial orbits for the reasons just described. Finally, applications of LEO constellations generally require coordinated use of the satellites, meaning operators prefer to have temporal and spatial symmetries across their constellations. Nevertheless, a particular shell might be shared by many small constellations (demonstrating little shell-wide symmetry), occupied by a single large constellation (with high temporal and spatial shell symmetry), or be somewhere in the middle.

\subsection{Determination of the minimum distance between satellites}

The study of minimum distance between satellites at any time in their dynamic is one of the most costly computations that must be done in order to assure the slotting requirements. In that regard, Speckman et al. (1990) has provided an important analytical result to ease this calculation. In that study the closest (non-dimensional) distance, $\rho_{\min }$, between two satellites in two circular orbits with same altitude is analytically expressed by the following equation:

$\rho_{\text {min }}=2\left|\sqrt{\frac{1+\cos i_{1} \cos i_{2}+\sin i_{1} \sin i_{2} \cos \Delta \Omega}{2}} \sin \left(\frac{\Delta F}{2}\right)\right|$,

where:

$\Delta F=\Delta M-2 \tan ^{-1}\left[-\tan \left(\frac{\Delta \Omega}{2}\right) \frac{\cos \left(\frac{i_{1}+i_{2}}{2}\right)}{\cos \left(\frac{i_{1}-i_{2}}{2}\right)}\right]$,

$i_{1}$ and $i_{2}$ are the orbit inclinations of the two satellites, and $\Delta M$ and $\Delta \Omega$ are the differences in mean anomaly and right ascension of ascending node of the two satellites, respectively. Note that $\rho_{\min }$ is a non-dimensional distance that must be scaled by the orbit radius to find the actual minimum approach distance value.

In this work, we will use a similar analytical expression that has been proven more computational efficient (Avendaño et al., 2020) when dealing with pairs of satellites located at any inclination:

$\alpha_{\min }=\frac{1}{2}\left|A+D+\sqrt{(A-D)^{2}+(B+C)^{2}}\right| ;$

where:

$$
\begin{aligned}
A= & \cos (\Delta \Omega) \cos (\Delta M)-\sin (\Delta \Omega) \cos \left(i_{1}\right) \sin (\Delta M) \\
B= & -\cos (\Delta \Omega) \sin (\Delta M)-\sin (\Delta \Omega) \cos \left(i_{1}\right) \cos (\Delta M) \\
C= & \cos \left(i_{2}\right) \sin (\Delta \Omega) \cos (\Delta M) \\
& +\cos \left(i_{1}\right) \cos \left(i_{2}\right) \cos (\Delta \Omega) \sin (\Delta M)+ \\
& \sin \left(i_{1}\right) \sin \left(i_{2}\right) \sin (\Delta M) \\
D= & -\cos \left(i_{2}\right) \sin (\Delta \Omega) \sin (\Delta M) \\
& +\cos \left(i_{1}\right) \cos \left(i_{2}\right) \cos (\Delta \Omega) \cos (\Delta M)+ \\
& \sin \left(i_{1}\right) \sin \left(i_{2}\right) \cos (\Delta M)
\end{aligned}
$$

where $\alpha_{\min }$ is the minimum angular distance between both satellites at any given instant. As it can be seen, the minimum distance provided by this equation is also nondimensional. These two equations are important results for those designing satellite constellations with circular 
orbits at the same altitude, since no propagation must be computed to check the minimum distance between each pair of satellites belonging to different orbital planes, which drastically reduces the computational time to assess this condition.

\section{Proposed approaches to shell design and results}

We proceed now to obtain solutions of $\left(B_{1}\right)$ using three different approaches. In all three cases, the goal is to maximize the number of satellites and Earth coverage of the whole configuration. To that end, we also discuss some insights regarding the results obtained from these different methodologies and comment on some key features regarding station-keeping of the configuration and adaptability to particular missions.

In our first approach, we propose a constellation of satellites chosen entirely at random that fulfills our slotting requirements. This kind of distribution will disregard the notion of symmetries altogether. Instead, this methodology results in a constellation with potentially as many satellites as different orbits, maximizing the number of orbits per shell and minimizing symmetries.

In the second approach, we focus on generating completely uniform distributions where satellites share the same inclination. To that end, we base the design of this kind of configuration on the 2D-LFC formulation. This way, we obtain highly symmetric solutions that have, in general, fewer orbits, and which are constrained to a particular inclination per altitude. It is important to note that although being restricted to a common inclination per shell could be seen as limiting from a design point of view, it minimizes the station-keeping necessary to maintain the overall configuration in the presence of the $J_{2}$ perturbation.

Our last approach is midway between the other two. The idea is to create the constellation as the union of different Flower Constellations. Following this process, we obtain solutions that share some of the characteristics of the previous methodologies. In this approach, we explore constellations defined at different inclinations and with a different numbers of satellites per Flower Constellation to explore the performance of this kind of distribution. A study on the optimal overlapping of two large Flower Constellations at different inclinations is also included.

In both the first and third approaches, we deal with slotting configurations whose satellites occupy different inclinations at the same altitude. Therefore, if no constraint is imposed in the propulsion system of those spacecrafts, these kind of solutions lead to unacceptably high stationkeeping costs to counter the $J_{2}$ effect (on the order of $100 \mathrm{~s}$ of $\mathrm{m} / \mathrm{s}$ per day). This is produced by the differential drift in the orbital planes of satellites that have different inclinations and limits the technical feasibility of this kind of solution. Nevertheless, it is important to note that these examples are useful in understanding limits to capacity in the absence of the $J_{2}$ effect and help inform trade-offs for other experiments involving a single or limited number of inclinations per shell.

As a side note, the astute reader will notice that we define the slotting problem in terns of the position, orientation, and shape of slots as a function of time but decline to present this information in this section. While we have generated these parameters, they are not particularly meaningful for the purpose of this paper, particularly for the stochastic approaches. Rather than the particular slots in a given solution, we are more interested in the performance of each approach to solution generation with respect to the four characteristics identified in the previous section. Table 1 shows a summary of the trade-offs of each approach studied in this work.

\subsection{Assumptions}

We make several assumptions across the experiments run in this section. Namely, we selected an altitude $h=700 \mathrm{~km}$ and $d_{\text {const }}=122 \mathrm{~km}$ (which corresponds to a minimum angular separation of 1 degree at all times). These parameters were chosen to enable comparability across the different methods. Nevertheless, $h$ and $d_{\text {const }}$ are free parameters that can and should be adjusted, either on a per-layer or constellation-wide basis as part of the design and optimization process. This is described in more detail in the current and next sections. For simplicity, we also assume spherical slots, although other slot geometries are possible and likely to be more efficient.

\subsection{Random constellation}

As a first case, we explore what would happen if satellites were placed into orbits at random within a shell, subject to the minimum-separation constraint. This roughly approximates what might occur if a rule were imposed to specify that orbits must be designed to avoid satellite vs. satellite conjunctions, but without other coordination.

Let us start by fixing some notation on orbital mechanics. We use the classical six orbital parameters $\mathcal{O}=\left(a, e, i, \omega, \Omega, M_{0}\right)$ to uniquely identify the trajectory followed by a satellite, assuming a keplerian model. Here $a$ is the semi-major axis, $e$ is the orbit eccentricity, $i$ is the orbit inclination, $\omega$ is the argument of the perigee, $\Omega$ is the right ascension of the ascending node, and $M_{0}$ is the mean anomaly at time $t=0$. The first five parameters provide the orbit of the satellite while $M_{0}$ locates the satellite within its orbit.

We call a "random constellation" to a solution of $\left(B_{1}\right)$ obtained by the following simple procedure:

$\left(R_{1}\right)$ Start with an empty constellation $\mathcal{C}=\varnothing$.

$\left(R_{2}\right)$ Find values of $i \in\left[i_{0}, i_{1}\right], \Omega \in\left[0^{\circ}, 360^{\circ}\right], M_{0} \in\left[0^{\circ}\right.$, $180^{\circ}$ ] uniformly at random, and let $s$ be the orbit whose elements are $\mathcal{O}_{s}=\left(R_{\text {earth }}+h, 0.0, i\right.$, $\left.0.0, \Omega, M_{0}\right)$; where $h$ is the altitude of the layer in which the constellation is to be placed. 
Table 1

Summary of approaches to shell design and trade-offs.

\begin{tabular}{|c|c|c|c|c|}
\hline & Number of Satellites & Earth Coverage & Different Orbits & Symmetries \\
\hline Random Constellation & Low & Targeted & Many & Few or None \\
\hline Single 2D-LFC & High & Uniform & Few & Many \\
\hline Union of 2D-LFCs & Medium & Varies & Intermediate & Intermediate \\
\hline
\end{tabular}

$\left(R_{3}\right)$ Check that $s$ is always more than $d_{\text {const }}$ apart from all the satellites in $\mathcal{C}$ at any time.

$\left(R_{4}\right)$ If the satellite fails the test, go back to $\left(R_{2}\right)$, unless we had $n_{\text {attempt }}$ consecutive fails, in which case we stop. Otherwise, add the satellite to $\mathcal{C}$ and go back to $\left(R_{2}\right)$.

The parameters $h$ and $d_{\text {const }}$ are given as input, and the output is the list of orbital elements of satellites. The number of trials, $n_{\text {attempt }}$, before terminating was hard-coded arbitrarily at 5 million. The intervals for $\Omega$ and $M_{0}$ were set to $\left[0^{\circ}, 360^{\circ}\right]$.

Table 2 shows the results of this study. The first two experiments use a range of inclinations, but as explained earlier satellites orbiting with different inclinations at a particular altitude will experience different rates of secular drift in the right ascension of the ascending node, imposing unacceptable frequent high $\Delta V$ to preserve alignment. These examples are included only to allow for comparison to the counter-factual case where $J_{2}$ was not present. In particular, the second experiment includes only prograde orbits, while the first includes both prograde and retrograde orbits. Generating the random constellation using just prograde orbits allows us to define a larger number of slots. This result is expected due to the different dynamics of prograde and retrograde orbits, which increase the potential intersections between the slots of the constellation. On the other hand, fixing the inclination of the constellation also reduces the maximum number of slots that the algorithm is able to generate. This is due to the additional constraint imposed in the configuration, which limits the freedom that the algorithm had originally to find empty locations. Finally, using close to equatorial and polar inclinations also reduces the number of available slots for the constellation. In this regard, $i=90^{\circ}$ is the worst case scenario due to the fact that all orbits intersect in the same points, the poles. This means that no matter the configuration used, it is not possible to improve the result of dis-

Table 2

Random constellation results.

\begin{tabular}{cc}
\hline Inclination $(\mathrm{deg})$ & Number of satellites \\
\hline$[0,180]$ & 1479 \\
{$[0,90]$} & 1910 \\
15 & 707 \\
30 & 927 \\
45 & 1015 \\
60 & 970 \\
75 & 850 \\
90 & 252 \\
\hline
\end{tabular}

tributing all satellites uniformly in the same polar orbit, that is locating 360 satellites under the considered conditions.

In order to show how these constellations are distributed in the space, the $(\Omega, M)$-space (Avendaño et al., 2013) (left) and $(\Omega, M)$-torus (Arnas et al., 2017c) (right) of one of these configurations are presented in Fig. 1. For this example we chose the solution for $i=60^{\circ}$ as a reference for its comparison with the other methodologies presented in this work. From Fig. 1 it can be observed that satellites are distributed quite randomly in the space, not generating clusters of satellites.

\subsection{Single $2 D-L F C$}

The idea behind this methodology is to assess all possible 2D-LFCs that fulfil the given criteria. In this regard, and contrary to what was studied in the work of Lee et al. (2015), we do not impose any additional constraints to the 2D-LFC. This means that the relative trajectory could be different for all the satellites of the constellation, and also that these relative trajectories may have selfintersections. At a first glance this conditions might appear to be a step back compared with previous methodologies. However, it turns out that constellations defined in relative trajectories with self-intersections provide the best solutions, not only in maximum number of satellites, but also in range of possible orbit inclinations.

Table 3 shows a summary of the results using 2D-LFC at various fixed inclinations. These results were obtained by searching for all possible 2D-LFC that have the selected inclination, whose number of orbits $\left(N_{o}\right)$ and number of satellites per orbit $\left(N_{s o}\right)$ were within the range from 1 to 360 , and that presented a minimum distance between satellites of at least 1 degree (which is assessed using Eq. (7)). This means that an exhaustive search on the possibilities of configuration that 2D-LFC provides is performed, where we selected configurations are the ones with a larger number of satellites for each inclination.

As can be seen in the results from Table 3, the maximum number of slots is heavily influenced by the inclination of the constellation. In fact, small variations in the inclination can produce noticeable changes in the maximum number of slots that can be defined. Similar to the random case, constellations whose inclinations are closer to $i=0^{\circ}$ and $i=90^{\circ}$ have lower capacity.

In addition, a complete study of all $2 \mathrm{D}-\mathrm{LFC}$ in the range of inclination $i \in\left[0^{\circ}, 90^{\circ}\right]$ in $0.1^{\circ}$ increments was performed in order to identify a best 2D-LFC candidate for this prob- 


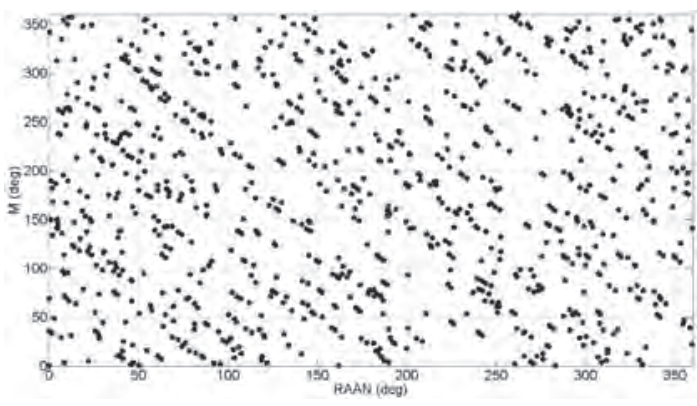

(a) $(\Omega, M)$-Space

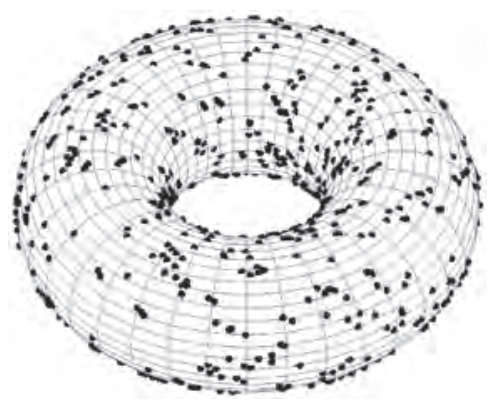

(b) $(\Omega, M)$-Torus

Fig. 1. Distribution of a Random Constellation for $i=60^{\circ}\left(N_{\text {sat }}=970\right)$.

Table 3

Single 2D-LFC results.

\begin{tabular}{|c|c|c|c|c|}
\hline \multirow[t]{2}{*}{ Inclination (deg) } & \multirow[t]{2}{*}{ Number of satellites } & \multicolumn{3}{|c|}{ Best Flower Constellation } \\
\hline & & $1 \leqslant N_{o} \leqslant 360$ & $1 \leqslant N_{s o} \leqslant 360$ & $0<N_{c}<N_{o}$ \\
\hline 15 & 1376 & 16 & 86 & 7 \\
\hline 45 & 1869 & 267 & 7 & 243 \\
\hline 60 & 1722 & 246 & 7 & 224 \\
\hline 75 & 1414 & 101 & 14 & 43 \\
\hline 90 & 359 & 1 & 359 & 0 \\
\hline
\end{tabular}

lem. To that end, no constraint in the parameters $N_{o}$ or $N_{s o}$ was imposed this time. Instead, the methodology used was the following $(\mathbf{L})$ :

$\left(L_{1}\right)$ Select a new inclination.

$\left(L_{2}\right)$ Set a number of satellites for the constellation $N_{\text {sat }}$.

$\left(L_{3}\right)$ Find the all pairs $\left\{N_{o}, N_{\text {so }}\right\}$ such that $N_{\text {sat }}=N_{o} N_{s o}$.

$\left(L_{4}\right)$ For each pair $\left\{N_{o}, N_{\text {so }}\right\}$ found, generate all possible satellite configurations varying $N_{c} \in\left\{0, \ldots, N_{o}-1\right\}$.

$\left(L_{5}\right)$ For each one of these constellation configurations, check the constraint of minimum distance between satellites:

- If the constraint is fulfilled, a constellation candidate for that number of satellites and inclination is generated. Continue in $\left(L_{7}\right)$.

- Otherwise, continue in $\left(L_{4}\right)$ with a different value of $N_{c}$.

$\left(L_{6}\right)$ Once all configurations have been checked for a given number of satellites, and if no configuration is found, we are sure that no uniform distribution can be defined that maintains the minimum distance constraint with that number of satellites and inclination. Then:

- If the process has changed the number of satellites more than 1000 times consecutively without finding a constellation that fulfills the minimum distance constraint, the process is finished for the studied inclination. Return to $\left(L_{1}\right)$.

- Otherwise continue in $\left(L_{7}\right)$.
$\left(L_{7}\right)$ Increase in one the number of satellites and return to $\left(L_{2}\right)$.

As a result of this study, the highest capacity was found at $i=46.2^{\circ}$ with a constellation of 2132 satellites. This constellation has the following 2D-LFC parameters: $N_{o}=2132, N_{s o}=1$, and $N_{c}=1772$. As can be seen, each satellite is located in a different orbital plane $\left(N_{s o}=1\right)$.

In general, the best solutions provided by the 2D-LFC formulation are defined in a large number of different orbital planes. Although this could be seen as a disadvantage of this kind of distribution, it is important to note that it is possible to distribute satellites in different right ascensions of the ascending node by taking advantage of the $J_{2}$ perturbation. This allows a single launch to distribute satellites to multiple planes while expending less fuel at the cost of some additional time after launch before reaching the desired operational orbit.

Compared with the results of random generated constellations, we observe that 2D-LFC are able to generate a larger number of slots for the same minimum distance between satellites and fixed inclinations. This effect is due to the natural structure and symmetries present in a $2 \mathrm{D}$ LFC. In particular, in order to assess the efficiency of 2D-LFCs, the following experiment was performed. First an optimal 2D-LFC was generated, like the ones presented in Table 3. Then, a numerical algorithm was created to include additional satellites in the configuration following the same process seen in Section 4.2. In all the tests performed at different inclinations and with different configu- 


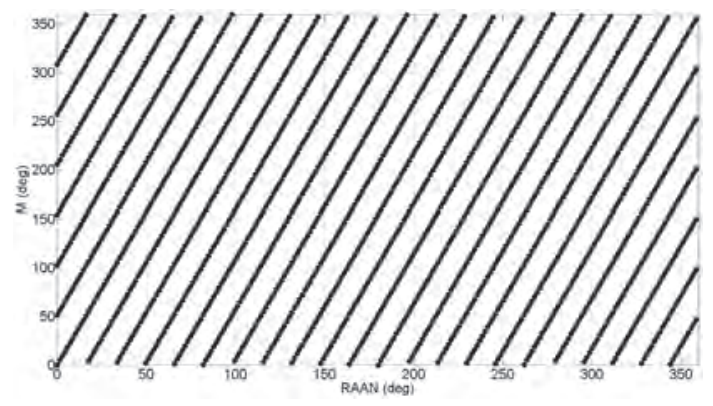

(a) $(\Omega, M)$-Space

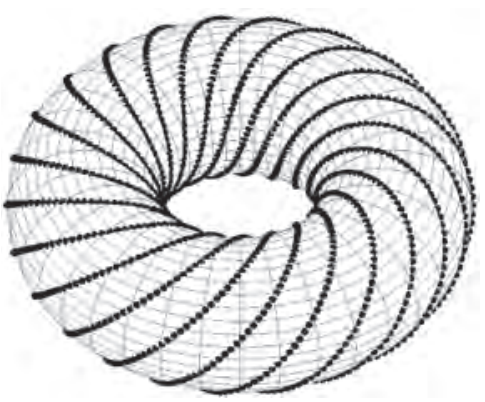

(b) $(\Omega, M)$-Torus

Fig. 2. Distribution of a Single Flower Constellation for $i=60^{\circ}\left(N_{\text {sat }}=1722\right)$.

rations, the algorithm was unable to include additional satellites. This means that 2D-LFCs are able to distribute satellites very efficiently, not leaving any empty space in their configuration.

Fig. 2 shows the $(\Omega, M)$-space distribution of a $2 \mathrm{D}$-LFC with parameters $N_{o}=246, N_{s o}=7$, and $N_{c}=224$. This distribution corresponds to the best 2D-LFC obtained at $i=60^{\circ}$ and shown in Table 3 . As it can be seen, the configuration is completely uniform. In particular, the relative distribution of the constellation remains identical no matter the satellite selected as the reference for the configuration.

Another interesting case of study is sun-synchronous constellations. A study of SSOs was performed for the chosen study parameters $(700 \mathrm{~km}$ of altitude implying $i=98.186^{\circ}$ and a minimum distance between satellites of $\left.1^{\circ}\right)$. Under these conditions, and using the methodology presented before $(\mathbf{L})$, the best $2 \mathrm{D}-\mathrm{LFC}$ found contains 1254 satellites, with constellation parameters: $N_{o}=418, N_{s o}=3$, and $N_{c}=160$. This result provides a upper boundary for the number of slots that it is possible to define for SSOs under the considered conditions.

Moreover, in order to evaluate the effect of varying the minimum distance between satellites in the overall capacity of the constellation, a more in-depth study was performed for SSOs. In particular, using 2D-LFC we assessed the maximum number of slots that can be defined as a function of the minimum distance between satellites. This computa- tion was performed following the same procedure described in $(\mathbf{L})$. The results from this study can be seen in Fig. 3. From it, we can observe that the relation between minimum angular distance and maximum number of slots is linear when expressing it in logarithmic scale. Therefore, it is reasonable to expect the maximum number of satellites to scale, in order of magnitude, with a function of the form $N_{\text {sat }}=A \alpha_{m}^{-B}$, where $N_{\text {sat }}$ is the maximum number of slots that can be defined, $\alpha_{m}$ is the minimum distance between satellites, and $A$ and $B$ are two constants. This result seems also to be coherent with a uniform distribution of the area of a sphere between $N_{\text {sat }}$ points if we consider $\alpha_{m} \ll 1$ :

$N_{s a t} \sim \frac{4 \pi R^{2}}{2 \pi R^{2}\left(1-\cos \left(\frac{\alpha_{m}}{2}\right)\right)} \sim \frac{16}{\alpha_{m}^{2}+o\left(\alpha_{m}^{3}\right)} \sim \frac{16}{\alpha_{m}^{2}}$

where $R$ is the radius of the sphere, and the two related areas are the complete surface of the sphere, and the area of a spherical casket of radius $\alpha_{m}$. Thus, we can conclude that the maximum number of satellites depends heavily on the size of the control box and the inclination, with more sensitivity to the size of the control box.

\subsection{Union of $2 D-L F C s$}

In this subsection we focus on the study of combining different 2D-LFCs in a single shell instead of just one 2D-LFC. To that end, three different approaches have been

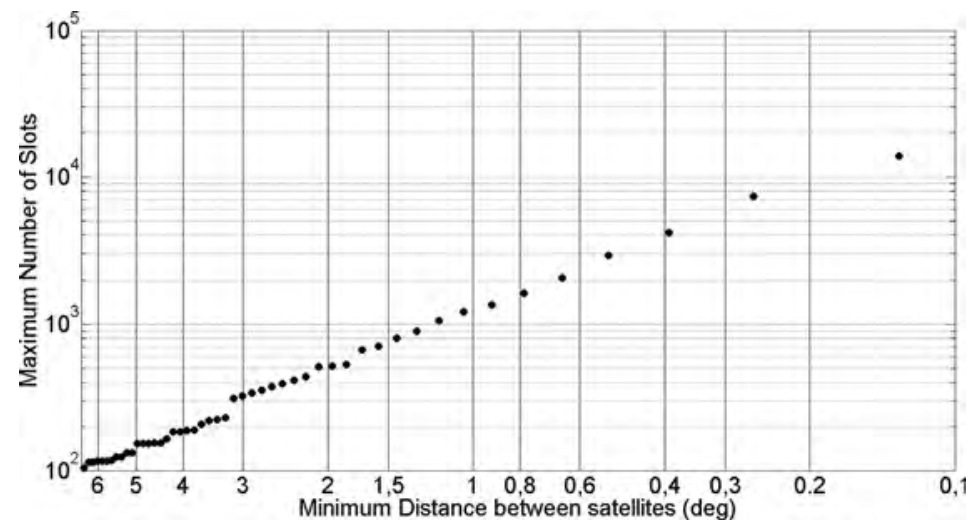

Fig. 3. Maximum number of slots in sun-synchronous orbits (based on the 2D-LFCs) as a function of the minimum angular distance between satellites. 
performed to cover a wide range of configuration possibilities. Since these solutions depend heavily on the random configurations generated, the results obtained should be regarded as reference values for similar studies, not as a complete general result.

\subsubsection{Random union of orbits}

In the first approach, the idea is to merge different 2DLFCs that are generated at random. The 2D-LFCs selected for this approach are limited to constellations defined in a single orbit, the most simple 2D-LFC that can be generated. Therefore, the process is similar to the one presented for the random constellations (see Section 4.2), but instead of just including one satellite in each iteration of the algorithm, a set of satellites uniformly distributed in the randomly generated orbit are computed and checked under the minimum distance constraint. If all the satellites in that orbit fulfil the condition, this subset of satellites is included in the constellation. Otherwise, the process is repeated. This method could represent a scenario where satellites are launched in groups to the same inertial orbit to reduce launch costs.

Table 4 shows the results for this approach. As can be seen, all these configurations perform better than their equivalents using a pure random approach (see Section 4.2). This is due to the fact that providing a structure for the constellation, even if it is as simple as the one proposed in this approach, allows it to better optimize the configuration space. This effect is more noticeable when using pure distributions based on the Flower Constellations formula-

Table 4

Random union of orbits results.

\begin{tabular}{ccc}
\hline Satellites per Orbit & Inclination $(\mathrm{deg})$ & Number of Satellites \\
\hline 2 & {$[0,180]$} & 1580 \\
3 & {$[0,180]$} & 1638 \\
4 & {$[0,180]$} & 1676 \\
5 & {$[0,180]$} & 1770 \\
6 & {$[0,180]$} & 1692 \\
10 & {$[0,180]$} & 1800 \\
15 & {$[0,180]$} & 1665 \\
20 & {$[0,180]$} & 1620 \\
30 & {$[0,180]$} & 1710
\end{tabular}

tion, since they represent the most structured configurations.

Fig. 4 shows the $(\Omega, M)$-space (left) and the $(\Omega, M)$-torus (right) of the solution for 10 satellites per orbit and 1800 maximum number of slots presented in Table 4. As it can be seen, orbits (vertical lines in the figure) are deployed quite uniformly in the space, more than in the completely random approach (Fig. 1) but much less than in the 2DLFC approach (Fig. 2). Note that these orbits are defined at different inclinations, and thus, these figures only represent the projection of the configuration in the $(\Omega, M)$-space.

\subsubsection{Random union of $2 D-L F C s$}

In this set of experiments, we explore generating sets of overlapping random 2D-LFCs, each including a subset of satellites uniformly distributed following the 2D-LFC formulation (see Eq. (1)). As in previous cases, satellites are generated and checked under the minimum distance constraint in each algorithm iteration. If all the satellites in that 2D-LFC fulfil the condition, this subset of satellites is included in the constellation; otherwise, the process is repeated. In this case, we study both the situation where satellites have a fixed inclination and when the inclination is left as a free parameter for the search algorithm. A summary of the results of both studies can be seen in Tables 5,6 .

From the results obtained we can see that this strategy has less capacity than the single 2D-LFC. Interestingly, capacity was not monotonic with respect to number of satellites per Flower Constellation for most inclinations, meaning that there are situations where it is beneficial for the algorithm to have more degrees of freedom during the searching process. During this study no condition was found to determine when these situations happen.

\subsubsection{Combination of two $2 D-L F C$ at different inclinations}

In this third approach, we generated two large 2DLFCs, each one with $N_{o}=986, N_{s o}=1$, and $N_{c}=478$, at different inclinations, $i=\left\{40^{\circ}, 60^{\circ}\right\}$. Each $\mathrm{FC}$ has 986 satellites for a total capacity of 1972 satellites. The chosen constellations were computed with compatible phasing and checked under the minimum distance constraint. As can be seen, this result achieves capacity comparable to a pure 2D-

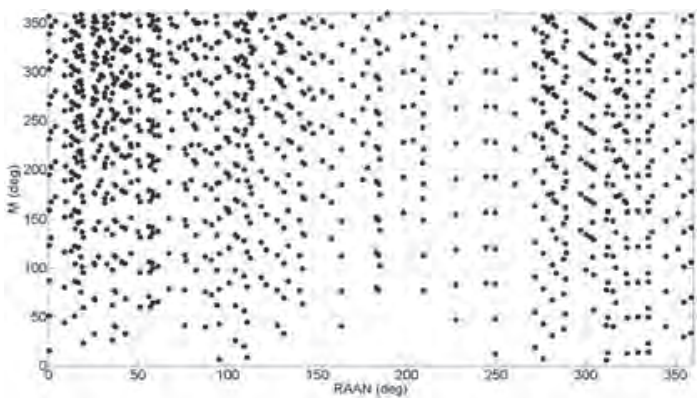

(a) $(\Omega, M)$-Space

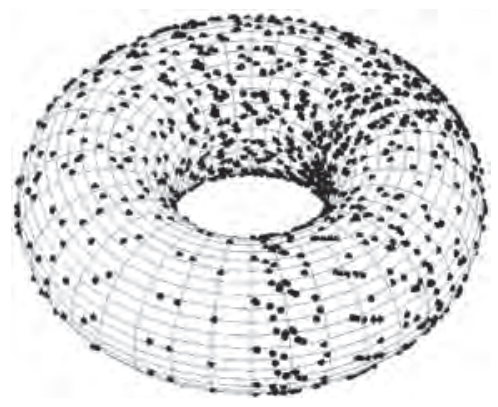

(b) $(\Omega, M)$-Torus

Fig. 4. Distribution of a Random Union of Orbits with $N_{s o}=10\left(N_{\text {sat }}=1800\right)$. 
Table 5

Union of 2D-LFCs Results $(i \in[0,180])$.

\begin{tabular}{ccc}
\hline $\begin{array}{c}\text { Satellites per Flower } \\
\text { Constellation }\end{array}$ & $\begin{array}{c}\text { Inclination } \\
(\mathrm{deg})\end{array}$ & $\begin{array}{c}\text { Number of } \\
\text { Satellites }\end{array}$ \\
\hline 2 & {$[0,180]$} & 1182 \\
3 & {$[0,180]$} & 1287 \\
4 & {$[0,180]$} & 876 \\
5 & {$[0,180]$} & 1460 \\
6 & {$[0,180]$} & 1068 \\
10 & {$[0,180]$} & 960 \\
15 & {$[0,180]$} & 960 \\
20 & {$[0,180]$} & 1080 \\
100 & {$[0,180]$} & 1000 \\
\hline
\end{tabular}

Table 6

Union of 2D-LFCs Results (fixed inclination).

\begin{tabular}{ccc}
\hline $\begin{array}{c}\text { Satellites per Flower } \\
\text { Constellation }\end{array}$ & $\begin{array}{c}\text { Inclination } \\
(\mathrm{deg})\end{array}$ & $\begin{array}{c}\text { Number of } \\
\text { Satellites }\end{array}$ \\
\hline 5 & 30 & 845 \\
10 & 30 & 760 \\
20 & 30 & 820 \\
5 & 45 & 815 \\
10 & 45 & 770 \\
20 & 45 & 780 \\
5 & 60 & 755 \\
10 & 60 & 770 \\
20 & 60 & 860 \\
5 & 75 & 695 \\
10 & 75 & 660 \\
20 & 75 & 700 \\
\hline
\end{tabular}

LFC. This shows the potential of this kind of configuration. Note that this kind of distribution has the problem of presenting a differential drift in the orbital planes due to the different inclinations. This would require, in general, prohibitively large and frequent station-keeping to preserve this configuration.

Fig. 5 shows the resultant constellation. In it, two different subsets of satellites are presented corresponding with inclinations of $i=40^{\circ}$ and $i=60^{\circ}$ respectively. As can be seen, the overall configuration of the constellation in the right ascension of the ascending node and the mean anomaly is in fact a $2 \mathrm{D}-\mathrm{LFC}$.

\section{Discussion}

This section describes the various free parameters available in the trade-space of our analysis model. These factors are parameters that can be set based on both engineering requirements and policy guidance, with consequences for system design and capacity.

\subsection{Minimum separation distance}

As seen from the results obtained in Section 4, the minimum distance between slots is the most important parameter to determine the maximum number of slots in a given shell, followed by the inclination of the orbits. This means that small changes in the minimum distance between satellites lead to large variations in the maximum number of possible slots of the configuration.

For the analysis in this paper, we have chosen a deliberately conservative 1 degree separation, which at the chosen $700 \mathrm{~km}$ altitude corresponds to approximately $122 \mathrm{~km}$. Similar studies have been made for other angular separations. An example of that can be seen in Fig. 3. It is clear that smaller values allow denser packing of traffic, but reduce distances between spacecraft and may require improved state knowledge on the part of operators and more frequent maneuvering to resist perturbations. A different value could be chosen either for the entirety of LEO, or for a certain altitude. In particular, we should expect to loosen the minimum distance requirement as the altitude of the orbit increases. This is mainly due to two effects. First, higher orbits are perturbed less by orbital perturbations such as the atmospheric drag or the $J_{2}$ term of the Earth's gravitational field. Second, the dynamic of higher orbits is slower when compared to lower orbits. This means that we should expect a larger maximum number of slots in shells at higher altitudes, and a lower number at lower altitudes.

In addition, it is important to note that using a slotting methodology it is still possible to perform formation flying between satellites. In these cases, multiple satellites are expected to operate in a single or various slots, with the operator being responsible for the control of the formation inside the slot boundaries. This concept is similar to how

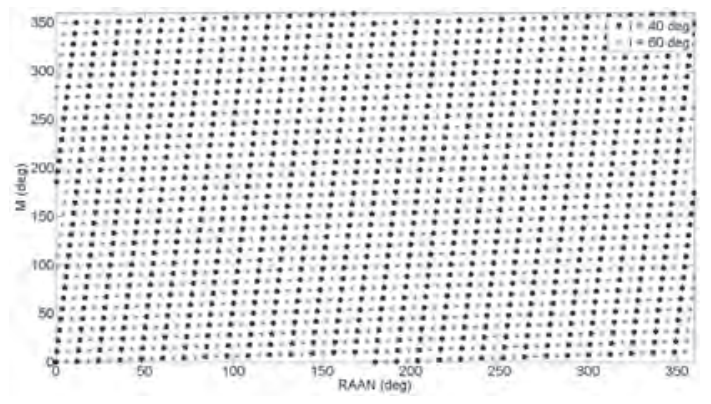

(a) $(\Omega, M)$-Space

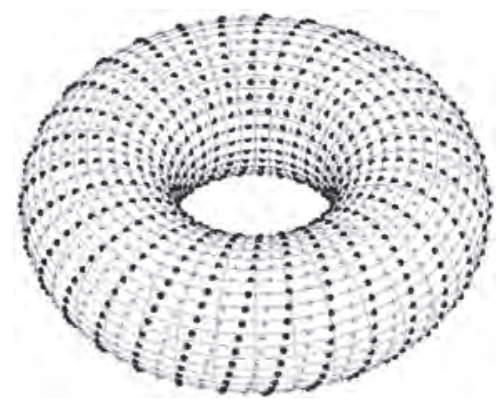

(b) $(\Omega, M)$-Torus

Fig. 5. Distribution of a Union of Two 2D-LFCs $\left(N_{\text {sat }}=1972\right)$. 
multiple satellites can share overlapping physical slots at GEO.

Another important topic to describe is the actions available to an operator should a conjunction occur between a slotted satellite and a non-compliant satellite or debris object. This area is still a work in progress, but the potential options that are being considered are maneuvering within one's slot if possible, or using a set of empty "street" shells between parking orbits for inter or intra shell maneuvers (see also next subsection).

\subsection{Layer altitude separation}

In general, layer altitude separation should take into account the effects of at least the $J_{2}$ orbital perturbation and atmospheric drag. This means that layer altitude separation is heavily influenced by accuracy of satellite state knowledge and size of the control box defined for each shell. Therefore, a looser control box for layer altitude separation would allow a longer period of time between station-keeping maneuvers, but result in lower overall capacity. A preliminary analysis performed by the authors has shown that using a not very restrictive control strategy for satellites at $700 \mathrm{~km}$ of altitude, it is possible to separate layers at an approximate distance of $200 \mathrm{~m}$ with monthly station-keeping. Drag effects are smaller at higher altitudes and may permit even closer spacing. At lower altitudes, wider spacing between slots would be required.

Additionally, and in order to allow flexibility and some mobility inside the slotting configuration, we propose to leave some shells completely empty. These shells are included to facilitate:

- The reconfiguration of the constellation,

- Possible orbital maneuvers between slots,

- Some parking capabilities,

- Additional room in case collision avoidance maneuvers are needed to mitigate conjunctions with noncompliant satellites or debris objects,

- And a limited adaptation of the configuration to accommodate some elliptic orbits.

This means that, following this design, we should expect an empty layer between each two pairs of occupied shells. This idea reduces the capacity of the system, but it provides a lot of flexibility for the slotting configuration.

\subsection{Inclination}

Section 4 shows that the inclination of the orbits in a shell is a key parameter that affects the maximum number of slots that can be defined. In that regard, we were able to obtain the maximum number of slots for a shell, under a 1 degree minimum separation between satellites, at an inclination of $i=46.2^{\circ}$. Note that this inclination is not unique. There is a range of inclinations that fulfill the previous condition, a situation that should be used to define a safe dynamic under orbital perturbations. While this inclination offers maximum capacity among the experiments we explored, it may or may not be suitable for a particular application due to a lack coverage of higher latitudes. Higher inclinations, including sun-synchronous orbits, can be accommodated, but at a reduction in the total number of slots that can be supported in that shell. In particular, orbital planes close to an equatorial or polar configuration significantly reduces the number of satellites per shell, with $i=0^{\circ}$ and $i=90^{\circ}$ being the worst solutions for capacity.

We have already discussed the problem of defining shells whose slots have different inclinations. Having shells with slots at different inclinations allows more flexibility in constellation design, but imposes very important limitations on satellite propulsion systems, being technically unfeasible in many cases. Shells with a common inclination in their orbits have a reduced cost for orbit maintenance. For that reason, a special focus has been made to explore singleinclination shells. Unfortunately, the need to enforce a single inclination per shell is the most burdensome implication of this slotting system. Nevertheless, it can be somewhat mitigated by the use of large numbers of nested circular altitude shells.

\subsection{Shell generation strategy}

In Section 4, we discuss three different potential strategies to generate orbits within a shell. Overall, the random constellation approach offers the most flexibility regarding satellite placement, but is only about $50-70 \%$ efficient as compared to a single Flower Constellation approach. This random strategy is probably the most similar to the current approach and future trend if no measures are taken, accommodating traffic on an ad-hoc basis without committing to certain constellation structures.

On the other hand, a union of small Flower Constellations shows that multiple Flower Constellations perform significantly worse than a single Flower Constellation and are only moderately sensitive to the number of satellites per orbit. Nevertheless, this kind of distribution shows that having a structure in the constellation, even a basic one, provides an improvement in the capacity of the system.

All else being equal, a single 2D-LFC provides greater capacity, better coverage, and a maximum number of symmetries. In that regard, Fig. 6 shows an example of the distribution of this kind of constellation from an Earth perspective. As can be seen, satellites uniformly cover all the regions of the Earth between the latitudes defined by their inclinations (in this example $60^{\circ}$ ). Therefore, among the single-inclination shell generation approaches considered in this paper, 2D-LFCs show the best packing. In particular, a single $2 \mathrm{D}-\mathrm{LFC}$ is especially appropriate when actors have compatible mission requirements (Arnas, 2018) and the chief objective is to maximize capacity, or if a shell's primary user is a single large constellation. This 


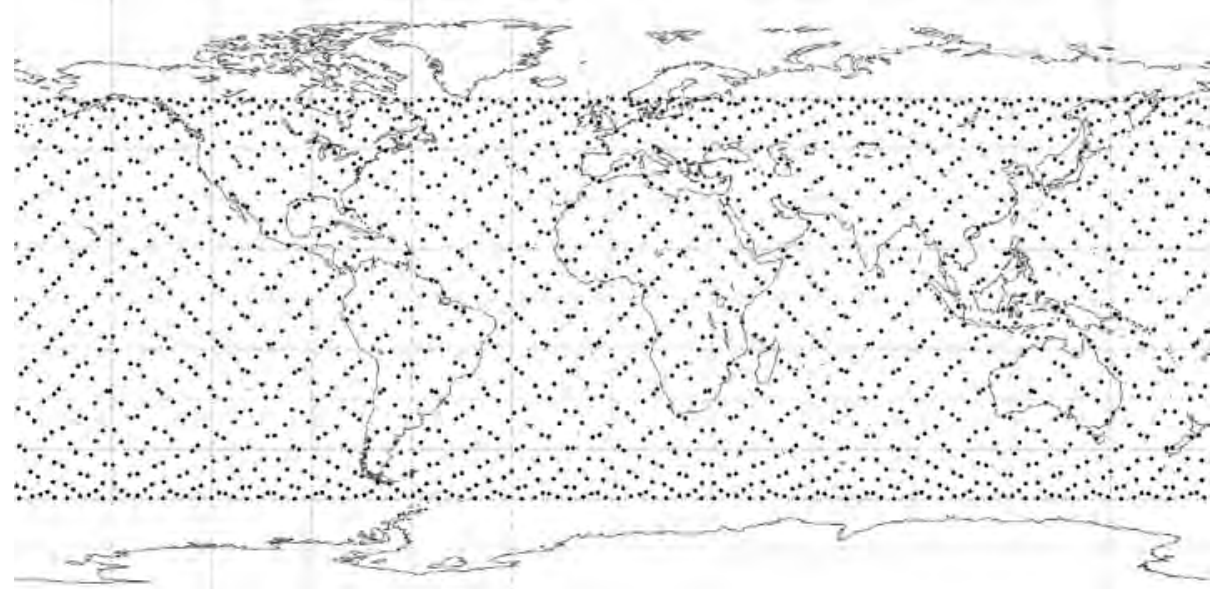

Fig. 6. Distribution of a 2D-LFC $\left(i=60^{\circ}, N_{o}=246, N_{s o}=7\right.$, and $\left.N_{c}=224\right)$ from an Earth perspective.

shows that constellation design models based on the Flower Constellation Theory (Mortari et al., 2004; Avendaño et al., 2013; Arnas et al., 2017a; Arnas et al., 2017b) are an adequate tool to study this problem.

\subsection{Adaptation of the slotting systems to current satellites in orbit}

The prior analysis does not take into account that there are many resident space objects already in orbit, some active and many inactive. Ideally, an orbital slotting system would allow for the retroactive integration of these active satellites into a proposed slotting system to minimize burden on their operations and maximize the benefits to existing and future spacecraft.

Inactive objects do not station-keep and cannot be integrated. Conjunctions with non-compliant objects (both active and inactive) would need to be analyzed using procedures similar to those used today for maneuver coordination for conjunctions between active objects. The empty phasing shells could potentially be used for collision avoidance maneuvers, or collision avoidance maneuvers could be conducted within a particular parking slot if station knowledge is sufficiently precise for both objects. The authors intend to explore both designing slots to optimize for existing active spacecraft and defining conceptions of operation for collision avoidance under an orbital slotting system in future work. Integration of satellites in near circular orbits, the vast majority of active satellites, is significantly simpler than satellites in eccentric orbits which would require further theoretical development and case by case study.

In general, the same checking procedure used for the random generation example in Section 4.2 and the union of small random 2D-LFCs in Section 4.4.2 could be used to generate slots compliant with existing objects. Under this checking approach, generating shells compatible with existing objects is limited to objects that are already in compatible orbits with one another, i.e. sets of orbits that do not result in hazardous self-conjunctions. When multi- ple objects are in overlapping orbits with non-compatible orbits, the shell design can only be optimized for a compatible subset of such objects. At altitudes where a large constellation is designed to avoid self-conjunctions, additional slots could be generated in compatible orbits. For altitudes where there are many satellites in orbits that are not designed to avoid conjunctions, the room for optimization may be more limited.

Some room to accommodate existing objects also exists when using the 2D-LFC shells. 2D-LFCs are defined based on a relative distribution of the slots. This means that it is possible to define the slotting architecture in each shell based on the objects already in orbit, selecting an appropriate slot distribution to avoid conflict with the dynamics of previous missions. In addition, the inclination of the 2DLFC can be chosen to minimize interference with prior traffic or offer the best trade-off between interference with existing traffic and demands for new satellites to be placed into the shell.

These methods allow designers to adapt a proposed architecture to minimize interference with current active objects in orbit at a cost to the designer's flexibility in slot definition and/or overall system capacity.

\subsection{Overall capacity estimate}

In this section we estimate a potential number of admissible slots in LEO. We caution that this estimate is heavily dependent on the assumptions for the above free parameters and the selection of which approach and parameters are used for each layer. In particular, including more sun-synchronous shells, or clearing additional slots to accommodate elliptical orbits, "street" layers, or launch corridors will reduce the total from the value we reach. Rather than the specific value obtained in this estimate, this section is included primarily to demonstrate that it is possible to generate large numbers of slots in LEO that avoid self-conjunction. Moreover, the process followed to obtain this estimate illustrates a clear tool to conceptualize LEO 
capacity and trade-offs between capacity and specific uses of orbital regions (for instance, the inclination selected for a particular shell).

For this estimate, we assume that the shells start at $650 \mathrm{~km}$ and end at $2000 \mathrm{~km}$, with occupied layers every $1 \mathrm{~km}$ (this provides sufficient space for an empty layer between every two occupied layers and some additional safety margin). This gives us a total of 2700 layers, 1350 of which are occupied. In addition, if we assume a global minimum distance between satellites of 1 degree (that is, $d_{\text {const }}$ does not depend on the altitude of the shell), we have estimated an average of 1700 slots per shell. This means that under this conditions, it is possible to define a total of 2.3 million admissible slots in the LEO region. Note that this value is a conservative estimate that is expected to greatly vary depending on the minimum distance allowed between satellites and the final configuration selected. For instance, if one were to assume every shell had the same capacity as a $700 \mathrm{~km} \mathrm{SSO}$ shell, ${ }^{2}$ this would yield nearly 1.7 millions satellites. In contrast, if a per-satellite separation of 0.13 degrees were used, this number would jump to nearly 18.7 million admissible slots.

\section{Conclusion}

In this paper we briefly described the motivation for a LEO slotting system, presented a formulation of the problem, and showed three approaches for slot generation and their performance across a set of four metrics. We then described several of the parameters available to the designer of a potential Space Traffic Management LEO slotting system and studied their impact on the performance of the system, with a special focus on system capacity.

The results obtained in this study show that if no policy measure is defined or norm emerges for the LEO STM slotting problem, the resultant distribution of the space in LEO will be highly sub-optimal, clearly benefiting the first operator to launch a significant number of satellites at a given altitude. Even with very simple slotting strategies, the capacity of the system, as well as its long term maintenance, improves significantly. For instance, we show that using random 2D-LFCs instead of a set of randomlyplaced single slots provides benefits not only in overall capacity, but also in orbit maintenance. The upper limit of this structured configurations is set by the 2D-LFCs. This formulation allows the generation of completely uniform distributions that present the maximum number of symmetries in their configuration. 2D-LFCs support the largest number of satellites among the methodologies studied, and also allow the easiest control under the effect of orbital perturbations.

\footnotetext{
${ }^{2}$ Even if only SSO orbits were used, inclination and thus capacity will vary slightly with altitude.
}

Additionally, we carried out a first study on the overall capacity of the slotting system. In particular, given a particular altitude, the authors were able to locate 2132 satellites in circular orbits while maintaining a minimum separation between satellites of 1 degree during their whole dynamic. In general, and for shells of satellites at the same altitude and inclination, the maximum number of slots that can be defined is a direct function of the minimum distance allowed between satellites and the inclination of the orbits, where the minimum distance allowed between satellites is the most decisive parameter.

The concept proposed in this work is still in its early stages of analysis. Some areas for additional technical work include theoretical expansions (demonstration of the integration of high-value elliptical orbits, other non-spherical Earth effects, incorporation of corridors for launch and orbit phasing, control-box analysis and sizing); development and simulation of concepts of operation for common orbital behaviors within the slotting structure (phasing, rendezvous and proximity operations, transfer orbits, reentry, servicing, etc.); optimization (alignment of shells to minimize the impact of current debris and satellite activities, analysis of different allocation policies, resiliency to orbital failures and non-compliant satellites); and further metrics development and cost-benefit analysis (including design reference missions and evaluation criteria, comparison of station-keeping costs and capacity against constellation designs that do not guarantee non-intersection, integrated capacity-risk metrics). We actively invite others within the community to develop other potential approaches and solutions that satisfy the LEO slotting problem.

We also invite feedback from those within the policy community. The technical feasibility of LEO slotting is a necessary, but not sufficient condition for the implementation of such a system. Considerable thinking is required to decide if such a system is desirable. We acknowledge that there are important considerations including slot allocation, shell-design optimization strategies, registration, and coordination/ enforcement that we have not addressed in this paper. Ongoing discourse between technical and policy experts will be critical to help mature the proposed concept, characterize stakeholder requirements and preferences, and ensure any such slotting system is fair, equitable, efficient, and responsive to stakeholder needs.

\section{Acknowledgments}

The authors wish to acknowledge useful conversations with Prof. Daniele Mortari at Texas A\&M University on Flower Constellation Theory.

David Arnas was partially supported by grant ESP201787113-R (Spanish Ministry of Economy and Competitiveness). Martín Avendaño was partially supported by grant MTM2016-76868-C2-2-P (Ministry of Science and Innovation, Spain). All authors were also funded by MISTI Global Seed Funds (La Caixa Foundation). 


\section{References}

Alary, D., Giannopapa, C., Hedman, N., Hodgkins, K., Li, S., Murthi, K., Sanchez-Aranzamendi, M., Stubbe, P., Volynskaya, O., Wang, G., 2018. In: Schrogl, K.U., Jorgenson, C., Robinson, J., Soucek, A., (Eds.), Space Traffic Management - Towards a Roadmap for Implementation. International Academy of Astronautics, Paris, France.

Anilkumar, A., Aoyama, S., Baseley-Walker, B., Bilimoria, K., Curran, C., Drees, K., Giorgi, D., Greneche, E., Hetrick, R.B., Jing, M., Leshner, R.B., Liao, X., Maruyama, K., Marvao, R., Meijer, L., Michalski, X., Ono, A., Oprong, A.A., Pillokeit, S., Shortt, K., Smallhorn, T., Tegnerud, E., Ueta, A.Y., Tió, M.V., Weeden, B., Gao, W., Shen, W., Western, O.K., Zhang, G., Zavaleta, J., 2007. Space Traffic Management Final Report. Tech. rep. International Space University, Beijing, China.

Arnas, D., 2018. Necklace Flower Constellations (Ph.D. thesis). Universidad de Zaragoza.

Arnas, D., Casanova, D., Tresaco, E., 2017a. 2D Necklace Flower Constellations. Acta Astronaut. 142, 18-28.

Arnas, D., Casanova, D., Tresaco, E., 2017b. Time distributions in satellite constellation design. Celestial Mech. Dyn. Astron. 128 (2-3), 197-219.

Arnas, D., Casanova, D., Tresaco, E., Mortari, D., 2017c. 3-dimensional necklace flower constellations. Celestial Mech. Dyn. Astron. 129 (4), 433-448.

Arnas, D., Lifson, M., Linares, R., Avenda no, M., 2020. Low Earth Orbit slotting for Space Traffic Management using Flower Constellation Theory. In: AIAA Scitech 2020 Forum. p. 0721.

Avendaño, M., Arnas, D., Linares, R., Lifson, M., 2020. Efficient search of optimal Flower Constellations. arXiv prepint 2003.11970.

Avendaño, M.E., Davis, J.J., Mortari, D., 2013. The 2-D lattice theory of flower constellations. Celestial Mech. Dyn. Astron. 116 (4), 325-337.

Bilimoria, K.D., Krieger, R.A., 2011. Slot Architecture for Separating Satellites in Sun-Synchronous Orbits. In: AIAA Space, paper number 2011-7184. No. 2011-7184. American Institute of Aeronautics and Astronautics, Long Beach, CA.

De Sanctis, M., Rossi, T., Lucente, M., Ruggieri, M., Bruccoleri, C., Mortari, D., Izzo, D., 2008. Flower constellations for telemedicine services. In: Re, E.D., Ruggieri, M. (Eds.), Satellite Communications and Navigation Systems, Signals and Communication Technology. Springer, Boston, MA, pp. 589-598.

De Sanctis, M., Rossi, T., Lucente, M., Ruggieri, M., Mortari, D., Izzo, D., March 2007. Flower constellation of orbiters for martian communication. In: 2007 IEEE Aerospace Conference. IEEE, Big Sky, Montana, USA.

Draim, J.E., 1987. A common-period four-satellite continuous global coverage constellation. J. Guidance Control Dyn. 10 (5), 492-499.

Dufour, F., 2003. Coverage optimization of elliptical satellite constellations with an extended satellite triplet method. In: 54th International Astronautical Congress, paper number IAC-03-A.3.02. International Astronautical Federation, the International Academy of Astronautics, and the International Institute of Space Law, Bremen, Germany.

Gardner, R., Ostrom, E., Walker, J.M., 1990. The nature of common-pool resource problems. Rationality Soc. 2 (3), 335-358.

Jakhu, R.S., 2017. Regulatory process for communications satellite frequency allocations. In: Pelton, J.N., Madry, S., Camacho-Lara, S. (Eds.), Handbook of Satellite Applications. Springer, New York, NY, pp. 359-381.

Lee, S., Avendaño, M., Mortari, D., 2015. Uniform and weighted coverage for large lattice flower constellations. Adv. Astronaut. Sci. $156,3633-3648$.
Lee, S., Mortari, D., 2017. Design of constellations for Earth observation with intersatellite links. J. Guidance Control Dyn. 40 (5), 1261-1269.

Letizia, F., Lemmens, S., Bastida Virgili, B., Krag, H., 2019. Application of a debris index for global evaluation of mitigation strategies. Acta Astronaut. 161 (February), 348-362.

Luders, R.D., 1961. Satellite networks for continuous zonal coverage. ARS J. 31 (2), 179-184.

Marzano, F.S., Cimini, D., Memmo, A., Montopoli, M., Rossi, T., De Sanctis, M., Lucente, M., Mortari, D., Di Sabatino, M., 2009. Flower constellation of millimeter-wave radiometers for tropospheric monitoring at pseudogeostationary scale. IEEE Trans. Geosci. Remote Sens. 47 (9), 3107-3122.

McManus, L., Schaub, H., 2016. Establishing a formation of small satellites in a lunar flower constellation. J. Astronaut. Sci. 63 (4), 263-286.

Mortari, D., Davis, J.J., Owis, A., Dwidar, H., 2013. Reliable global navigation system using flower constellation. Int. J. Adv. Comput. Sci. Appl. 4 (2), 260-266.

Mortari, D., De Sanctis, M., Lucente, M., 2011. Design of flower constellations for telecommunication services. Proc. IEEE 99 (11), 2008-2019.

Mortari, D., Wilkins, M., Bruccoleri, C., 2004. The flower constellations. J. Astronaut. Sci. 52, 107-127.

Mortari, D., Wilkins, M.P., 2008. Flower constellation set theory part I: compatibility and phasing. IEEE Trans. Aerosp. Electron. Syst. 44 (3), 953-963.

Noyes, C.D., 2013. Characterization of the Effects of a Sun-Synchronous Orbit Slot Architecture on the Earth's Orbital Debris Environment (Master's thesis). California Polytechnic State University, San Luis Obispo, California.

OneWeb, 2019. Technology. (last accessed on December 1, 2019) https:// www.oneweb.world/technology\#video.

Park, K.J., Mortari, D., Ruggieri, M., 2005. Comparisons between GalileoSat and Global Navigation Flower Constellations. In: 2005 IEEE Aerospace Conference. IEEE, Big Sky, Montana, USA, pp. $1516-1523$.

Portillo, I., Cameron, B.G., Crawley, E.F., 2019. A technical comparison of three low earth orbit satellite constellation systems to provide global broadband. Acta Astronaut. 159, 123-135.

SpaceX, 2019. Starlink. (last accessed on December 1, 2019) https://www. starlink.com.

Speckman, L.E., Lang, T.J., Boyce, W.H., 1990. An analysis of the line of sight vector between two satellites in common altitude circular orbits. In: Astrodynamics Conference, 1990, Paper Number AIAA 90-2988CP. American Institute of Aeronautics and Astronautics Inc, AIAA, Portland, OR, pp. 866-874.

Telesat, 2019. Telesat LEO - Why LEO? (last accessed on December 1, 2019) https://www.telesat.com/services/leo/why-leo.

Walker, J., 1984. Satellite constellations. J. Br. Interplanet. Soc. 37, 559572.

Watson, E., 2012. Sun-Synchronous Orbit Slot Architecture Analysis and Development (Master's thesis). California Polytechnic State University, San Luis Obispo, California.

Weeden, B., Shortt, K., 2008. Development of an Architecture of SunSynchronous Orbital Slots to Minimize Conjunctions. In: SpaceOps 2008, paper number AIAA 2008-3547. pp. 1-15.

Weeden, B.C., Chow, T., 2012. Taking a common-pool resources approach to space sustainability: A framework and potential policies. Space Policy 28 (3), 166-172.

Wilkins, M.P., Mortari, D., 2008. Flower constellation set theory Part II: secondary paths and equivalency. IEEE Trans. Aerospace Electron. Syst. 44 (3), 964-976. 